\title{
Variational principle for steady states of dissipative quantum many-body systems
}

\author{
Hendrik Weimen* \\ Institut für Theoretische Physik, Leibniz Universität Hannover, Appelstr. 2, 30167 Hannover, Germany
}

(Dated: February 20, 2015)

\begin{abstract}
We present a novel generic framework to approximate the non-equilibrium steady states of dissipative quantum many-body systems. It is based on the variational minimization of a suitable norm of the quantum master equation describing the dynamics. We show how to apply this approach to different classes of variational quantum states and demonstrate its successful application to a dissipative extension of the Ising model, which is of importance to ongoing experiments on ultracold Rydberg atoms, as well as to a driven-dissipative variant of the Bose-Hubbard model. Finally, we identify several advantages of the variational approach over previously employed mean-field-like methods.
\end{abstract}

PACS numbers: 03.65.Yz, 02.70.Rr, 05.30.Rt, 32.80.Ee

From Fermat's principle of geometric optics, to Hamilton's principle of classical mechanics, to the maximum entropy principle of statistical physics, variational formulations are instrumental in our understanding of physical theories. Similarly, variational principles hold great power for the derivation of approximative solutions to computationally hard quantum many-body problems, as demonstrated by the successes of density functional theory [1] and the matrix product state formalism [2]. Here, we establish a variational principle for the steady states of dissipative quantum systems and demonstrate its effectiveness to tackle otherwise intractable problems.

Dissipative quantum many-body systems have recently received great attention, in particular following the discovery that dissipation can be a useful resource in the controlled preparation of quantum-many body states [314]. The interplay between coherent and dissipative dynamics can lead to a rich variety of novel physical phenomena, such as interaction-mediated laser cooling [15, 16], dissipative binding mechanisms [17, 18], and phase transitions in the non-equilibrium steady state of the dynamics 19 39]. However, the theoretical understanding of these systems is still fairly limited, especially because of the lack of a concept corresponding to the partition function in equilibrium systems, from which all quantities of interest can be derived. Additionally, dissipative quantum many-body system exhibit the same computational complexity as equilibrium systems, with the Hilbert space dimension growing exponentially with the size of the system.

In this Letter, we establish a variational principle for steady states of dissipative quantum many-body systems. Crucially, our approach is completely generic and allows to analyze arbitrary dissipative models using arbitrary variational quantum states. The key element is the minimization of a suitable norm for the underlying quantum master equation. We show how to evaluate this norm for different variational classes of correlated and uncorrelated density matrices and exemplify the application of our variational formalism using a dissipative
Ising model, which represents an important outstanding many-problem connected to experiments with strongly interacting Rydberg atoms. Importantly, our variational method is not limited to such Ising models, which we demonstrate by providing a variational analysis of the driven-dissipative Bose-Hubbard model. Finally, we conclude with a comparison of our variational approach to a previously employed method, establishing the advantages of our novel method.

The analysis of the stationary state is based on the quantum master equation describing the dynamics of the density matrix $\rho$. In the case of a Markovian evolution, it can be given in Lindblad form,

$$
\frac{d}{d t} \rho=-i[H, \rho]+\sum_{i}\left(c_{i} \rho c_{i}^{\dagger}-\frac{1}{2}\left\{c_{i}^{\dagger} c_{i}, \rho\right\}\right),
$$

where $H$ is the Hamiltonian describing the coherent evolution and the jump operators $c_{i}$ are associated with the incoherent part of the dynamics [40]. The stationary states of the system can then be obtained by solving the equation $d \rho / d t=0$. Outside of one-dimensional problems, where algorithms derived from the densitymatrix renormalization group can be applied [28, 29, 41], the exact stationary state cannot be computed for sufficiently large system sizes. In many previous works 24 26, 33, 34, 42 44, a mean-field approximation has been applied to study the stationary state. To understand the key features of our variational method, it is instructive to briefly restate the basic elements of this mean-field approach. There, the partial trace over the whole system except a single site is taken, resulting in the single site equations,

$$
\frac{d}{d t} \rho_{i}=\operatorname{Tr}_{i}\left\{\frac{d}{d t} \rho\right\}=-i\left[H_{i}, \rho_{i}\right]+\mathcal{D}_{i}\left(\rho_{i}\right) .
$$

where $H_{i}$ and $\mathcal{D}_{i}$ are the mean-field Hamiltonian and dissipative terms, respectively [45, 46]. The stationary state of these purely local master equations can be expressed as a product of single site density matrices, $\rho=\prod_{i} \rho_{i}$ 
and is found from the condition $d \rho_{i} / d t=0$ by solving the set of nonlinear equations.

Within our variational method, we take a different approach towards finding an approximate solution to the stationary state. Here, we take the full quantum master equation into account, i.e., without performing any partial trace operation. Instead, we approximate the true stationary state by a variational density matrix. Naturally, the true stationary state will in general be outside the space of possible density matrices that can be generated by a certain choice of variational degrees of freedom. As the crucial step, we approximate the true state by the variational state that minimizes a suitable norm of the full quantum master equation, i.e.,

$$
\|\dot{\rho}\|=\|-i[H, \rho]+\mathcal{D}(\rho)\| \rightarrow \min .
$$

At first glance, it might seem that the choice of the norm would be rather arbitrary without an underlying physical functional such as the (free) energy, but we will show that this is not the case. First, any suitable norm has to be invariant under unitary transformations of the form $\dot{\rho} \rightarrow U \dot{\rho} U^{\dagger}$, making it natural to focus on vector norms for the set of eigenvalues of $\dot{\rho}$ (e.g., Schatten norms such as the trace norm, Frobenius norm, or spectral norm). Second, the norm also has to be unbiased in the sense it does not favor certain classes of density matrices. For example, any Schatten norm not satisfying linearity according to $\|\dot{\rho}\|=\|\lambda \dot{\rho}\| / \lambda$ will, when applied to the time derivative of product states, always favor the maximally mixed state for sufficiently large system sizes [47]. These requirements single out the trace norm,

$$
\|\dot{\rho}\|=\operatorname{Tr}\{|\dot{\rho}|\} .
$$

In the following, we discuss how to evaluate this norm for different classes of variational density matrices.

Product states. - Similar to the mean-field approach, the simplest variational state one can construct is a product state of single site density matrices,

$$
\rho=\rho_{p} \equiv \mathcal{R} 1=\prod_{i} \rho_{i}
$$

where we have introduced the superoperator $\mathcal{R}$, which transforms every occurrence of the identity $1_{i}$ into the density matrix $\rho_{i}$. Then, the time derivative according to the quantum master equation has the form

$$
\dot{\rho}=\sum_{i} \mathcal{R} \dot{\rho}_{i}+\sum_{\langle i j\rangle} \mathcal{R} \dot{C}_{i j},
$$

where $C_{i j}$ is a matrix accounting for correlations between sites $i$ and $j$ and the double sum runs over all terms that are connected through interaction terms in the Hamiltonian or correlated jump operators. Note that although the variational state is a product state, the resulting dynamics will typically generate correlations. Here, we also see that the previous mean-field approach of solving the equation $\dot{\rho}_{i}=0$ only minimizes the first term in the norm $\|\dot{\rho}\|$, while the second term is uncontrolled.

At this point, a numerically exact minimization of the norm $\|\dot{\rho}\|$ is in general still a computationally intractable problem. However, it is vital to stress that our variational formulation represents the central step towards obtaining a good approximation to the stationary state. Importantly, for a successful application of a variational principle, we do not require an exact solution, but only to obtain an upper bound to the norm. Here, we can obtain such an upper bound through the inequality

$$
\|\dot{\rho}\| \leq \sum_{\langle i j\rangle} \operatorname{Tr}\left\{\left|\mathcal{R}\left(\dot{\rho}_{i} \rho_{j}+\rho_{i} \dot{\rho}_{j}+\dot{C}_{i j}\right)\right|\right\}=\sum_{\langle i j\rangle} \operatorname{Tr}\left\{\left|\dot{\rho}_{i j}\right|\right\},
$$

where we have used the reduced two-site operators $\dot{\rho}_{i j}=$ $\operatorname{Tr}_{i j j}\{\dot{\rho}\}$. Consequently, for a translationally invariant system, it is sufficient to minimize the norm $\left\|\dot{\rho}_{i j}\right\|$ of a single bond. Note that although only a single bond has to be treated exactly, the interaction with the other surrounding sites is still being accounted for on a meanfield level.

Interestingly, we point out that the solution to our variational approach depends on the coordination number $z$, in contrast to variational methods based on product states for equilibrium systems. Furthermore, in the limit $z \rightarrow \infty$, we recover the solution to the mean-field equation $\dot{\rho}_{i}=0$, which then also becomes the exact solution of the full quantum master equation.

Quantum and classical correlations. - We can now extend the class of variational states to go beyond product states. The first step in this direction is to include nearest-neighbor correlations of the form

$$
\rho=\rho_{c} \equiv \rho_{p}+\sum_{\langle i j\rangle} \mathcal{R} C_{i j}+\sum_{\langle i j\rangle \neq\langle k l\rangle} \mathcal{R} C_{i j} C_{k l}+\ldots
$$

subject to the constraint that all two-site density matrices $\rho_{i j}$ are positive, which guarantees the positivity of $\rho_{c}$ [46]. Using the same procedure as in the case of product states, we find

$$
\|\dot{\rho}\| \leq \sum_{\langle i j k\rangle}\left\|\dot{\rho}_{i j k}\right\|,
$$

i.e., the norm can be bounded from above by minimizing all combinations involving three sites. Note that on triangular lattices, it is necessary to distinguish three-site operators forming a loop from their open counterparts.

More generally, the variational approach also allows for basically arbitrary choices of density matrices, including long-range and higher-order correlations as well as longrange interactions. While the resulting variational functional will in general be more complicated than Eq. (9), the underlying variational principle will be unchanged. 


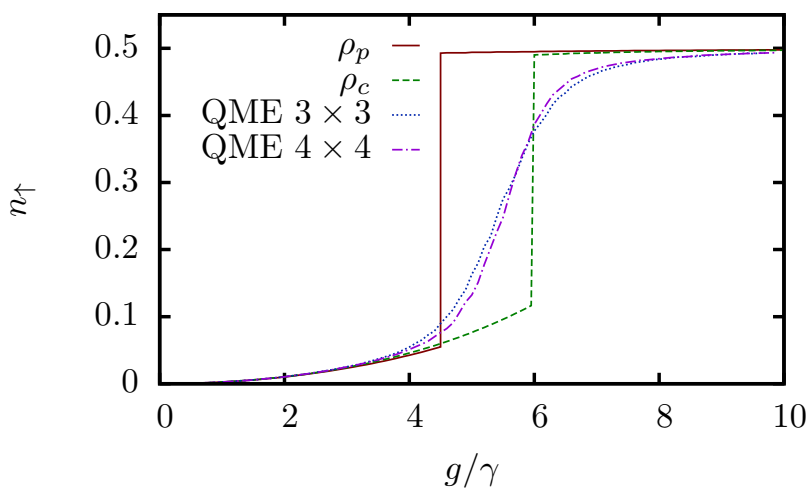

FIG. 1. Up-spin density $n_{\uparrow}$ in the steady state of the dissipative Ising model on a two-dimensional square lattice depending on the transverse-field $g$. Results are shown for the variational methods using product states $\rho_{p}$ and correlated states $\rho_{c}$, as well as solutions to the full quantum master equation (QME) for $3 \times 3$ and $4 \times 4$ lattices $(h=0, V=5 \gamma)$.

Dissipative Ising model. - We now turn to the study of a concrete model using our variational approach. We focus on a dissipative extension of the Ising model, in which one of the spin states is incoherently pumped into the other. This model has received considerable interest recently because of its expected relevance for ongoing experiments with ultracold Rydberg atoms [35, 37] and because of controversies surrounding the phase diagram of the nonequilibrium steady state [26, 29, 38, 49]. The quantum master equation of this model is given in the Lindblad form of Eq. (1), with the Hamiltonian following from an Ising model with interaction strength $V$, in both a transverse field $g$ and a longitudinal field $h$,

$$
H=\frac{g}{2} \sum_{i} \sigma_{x}^{(i)}+\frac{h}{2} \sum_{i} \sigma_{z}^{(i)}+\frac{V}{4} \sum_{\langle i j\rangle} \sigma_{z}^{(i)} \sigma_{z}^{(j)} .
$$

The jump operators describing spin flips can be expressed as $c_{i}=\sqrt{\gamma} \sigma_{-}$, with $\gamma$ being the decay rate of the up spins. In the context of experiments with Rydberg atoms, the down spin state corresponds to the atomic ground state, while the up spin state refers to a Rydberg state. The interaction strength $V=C_{6} / a^{6}$ follows from a repulsive van der Waals interaction described by a $C_{6}$ coefficient at the lattice spacing $a$ and transitions between the two states are driven by laser fields with a Rabi frequency $\Omega=$ $g$ and a detuning $\Delta$ that is related to the longitudinal field as $h=\Delta+z V / 2$ [50]. Finally, $\gamma$ refers to the radiative decay rate of the Rydberg state, which can be tuned by laser coupling to other excited non-Rydberg states [16].

For this model, the mean-field solution of Eq. (2) predict a region of bistability involving two distinct steady states over a large range of the transverse field $g$ 26, 38, 51]. This finding is contradicted by numerical simulations for one-dimensional systems based on the full quantum master equation [51, 52]. Here, our variational

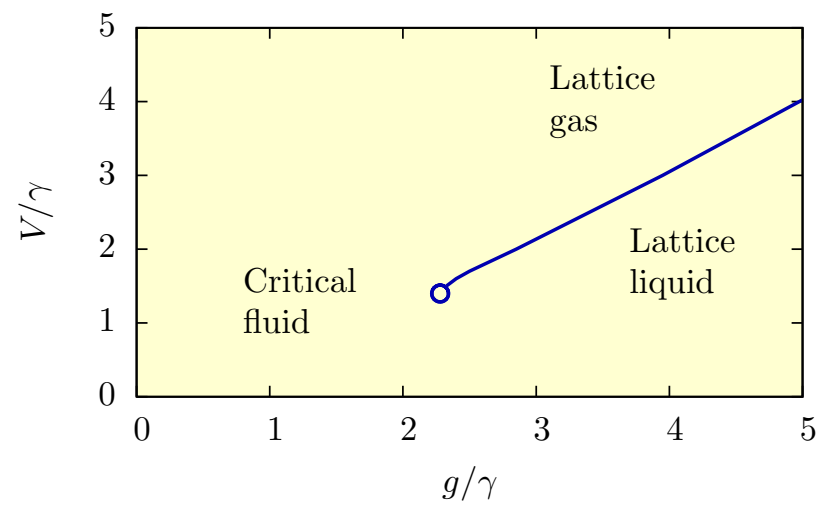

FIG. 2. Phase diagram of the dissipative transverse-field Ising model $(h=0)$. For large interaction strength $V$, there is a first-order transition between a lattice gas and a lattice liquid of up spins. This first-order transition ends in a critical point, around which the system forms a critical fluid.

approach agrees with the numerical results and always produces a unique steady state, both for product states $\rho_{p}$ and for correlated states $\rho_{c}$. On a two-dimensional square lattice, we find a first-order jump in the up-spin density $n_{\uparrow}$, see Fig. 1. Going from the variational product state $\rho_{p}$ to the correlated state $\rho_{c}$ results in a substantial increase of the critical value of the first-order transition, from $g_{c}=4.5$ to $g_{c}=6.0$.

We complement our variational approach with numerical simulations using a quantum trajectories method [53, 54] for $3 \times 3$ and $4 \times 4$ lattices with periodic boundary conditions. Due to finite-size effects, the first-order jump changes into a smooth crossover, and the position of the peak the susceptibility $\chi=\partial n_{\uparrow} / \partial g$ is shifted from its infinite-system value [55]. Here, we find the susceptibility peak at $g_{3 \times 3}=5.5$ and $g_{4 \times 4}=5.6$, respectively, implying good quantitative agreement between the numerical results and the variational procedure for correlated states, which is carried out in the thermodynamic limit of infinite system size.

Phase diagram. - We are now in the position to evaluate the phase diagram of the dissipative Ising model, see Fig. 2. Here, the quantum master equation does not exhibit any spin symmetry, as the dissipative terms break the $\mathbb{Z}_{2}$ symmetry of the Ising model even for $h=0$. Nevertheless, it is still possible to obtain phase transitions if an emergent symmetry arises, as in the well known case of the liquid-gas transition [56]. Here, it is natural to treat the up spins as particles, as then the dissipation can be understood as a particle loss process. Then, we have a similar transition from a low-density gas of up spins to a high-density liquid close to half filling with almost vanishing compressibility $\kappa=-\partial n_{\uparrow} / \partial h$ when the transverse field $g$ is increased over a critical value.

For weakly interacting particles, it is possible to apply perturbation theory in the interaction strength $V$, 
meaning that all observables are analytical and there is no phase transition. Therefore, it is naturally to associate the meaning of the inverse temperature $\beta$ in the liquid-gas transition to the interaction term $V$ in the dissipative Ising model. Similar identifications can also be made on the basis of the mean-field approach [38]. As it is the case in the classical liquid-gas transition, the variational approach based on correlated states predicts that the transition between the liquid and gas phases ends in a critical point, see Fig. 2, which we find here to be located at $(g, V)=(2.28,1.40) \gamma$.

Within our variational approach, we always obtain a unique solution for the stationary state and do not observe any regions of bistability. Close to the first-order transition, however, we observe two distinct states that both have a low residual dissipation and thus correspond to the bistable states obtained from the solution of the mean-field equations. Moreover, we would like to point out that the bistability observed in one experiment 35] could be explained by a hysteresis behavior close to firstorder phase transitions [57] (see also discussion in [38]).

Driven-dissipative Bose-Hubbard model. - To underline the extensive flexibility of our approach, let us turn to a driven-dissipative extension of the Bose-Hubbard model, which is equally paradigmatic as the quantum Ising model in the context of quantum many-body systems. Using bosonic creation and annihilation operators $b_{i}^{\dagger}$ and $b_{i}$, respectively, its Hamiltonian is of the form

$$
H=-J \sum_{\langle i, j\rangle} b_{i}^{\dagger} b_{j}+\sum_{i}\left[\frac{U}{2} n_{i}^{2}-\mu n_{i}+F\left(b_{i}+b_{i}^{\dagger}\right)\right]
$$

where $J$ describes the inter-site hopping of bosons, $U$ accounts for an on-site interaction involving the square of the density operator $n_{i}=b_{i}^{\dagger} b_{i}, \mu$ is the chemical potential of the particles, and $F$ describes a coherent driving term. The dissipative term follows from a single-particle loss of the bosons, which is described by jump operators of the form $c_{i}=\sqrt{\gamma} b_{i}$. The driven-dissipative Bose-Hubbard model has been discussed in a wide range of physical contexts, ranging from cavity and circuit QED arrays to exciton-polaritons in semiconductor microcavities 58 60].

Within our variational approach, we find a steady state solution for the boson density as shown in Fig. 3. We note that the variational phase diagram differs from the meanfield prediction [60] in several points: (i) The steady state is always unique, i.e., there is no bistability. (ii) There is a single first-order transition between a high-density phase at small values of the hopping $J$ and a low-density phase at large $J$. (iii) There are no features reminiscent of Mott lobes in the steady state.

Comparison with the mean-field solution. - Finally, we conclude by making a detailed comparison of our variational principle to the previously employed method of setting the mean-field equation of motion Eq. (2) to zero.

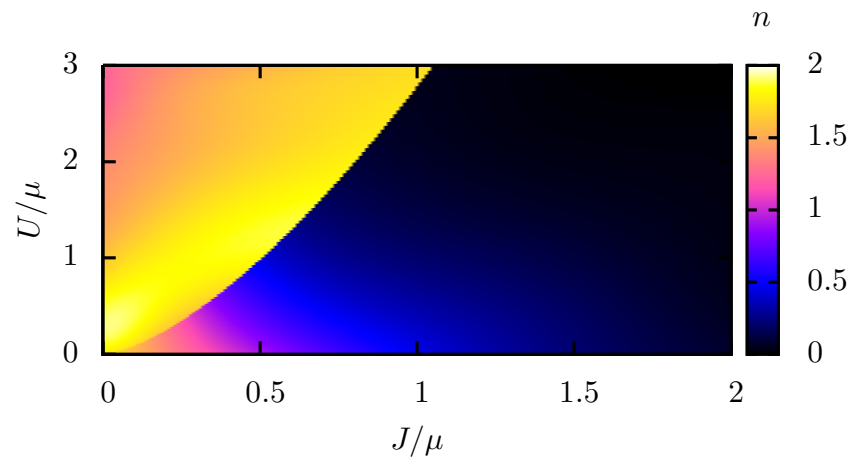

FIG. 3. Steady state density $n$ of the driven-dissipative BoseHubbard model $(F=0.4 \mu, \gamma=0.2 \mu)$. The system undergoes a first-order jump in the density when the hopping $J$ is increased over a critical value.

To ensure an unbiased comparison on an equal footing, we carry out the variational procedure based on product states $\rho_{p}$, as these states contain exactly the same free parameters as in the mean-field method. To be explicit, we focus on the case of the dissipative Ising model although the analysis can also be applied interchangeably to the Bose-Hubbard model.

As noted earlier, the mean-field solution predicts extended regions of bistable behavior, which is at odds both with numerical results and our variational procedure. But even outside the bistable region, there can be large qualitative and quantitative differences. In particular, we find these for large negative values of the longitudinal field $h$, where the mean-field solution predicts the existence of a large antiferromagnetic phase [26, 51]. Within our variational method, we find a drastic reduction of the extension of this phase. This finding is also in line with numerical simulations on a $4 \times 4$ lattice, which suggests at least a strong suppression (if not complete absence) of antiferromagnetic order.

More quantitatively, we can express the difference between the two methods by analyzing the norm $\left\|\dot{\rho}_{i j}\right\|$, expressing how well the respective solutions approach the true stationary state of the two-site dynamics. As expected, the variational method minimizing this norm results in it being small throughout the $g-h$ plane, see Fig. 4. On the other hand, we find much larger values for $\left\|\dot{\rho}_{i j}\right\|$ for the mean-field solution. In regions with $\left\|\dot{\rho}_{i j}\right\|>\gamma$, the contributions from the correlations $\dot{C}_{i j}$ in Eq. (6) are comparable to the spectral gap of the Liouvillian. This means the mean-field solution is even inconsistent as these terms generating correlations are of crucial importance and cannot be neglected as being only small perturbations.

In summary, we have established and successfully applied a novel variational principle for the non-equilibrium steady states of driven-dissipative quantum many-body systems. Besides the systems already studied in this 


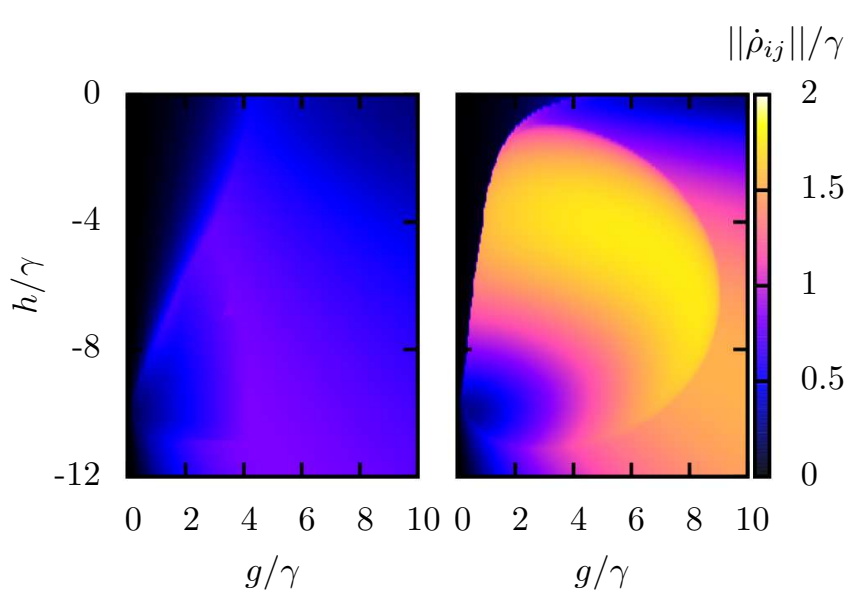

FIG. 4. Comparison of the norm of the two-site dynamics $\left\|\dot{\rho}_{i j}\right\|$ of the variational solution (left) and the mean-field solution (right) for the two-dimensional dissipative Ising model $(V=5 \gamma)$.

work, our method will be of importance to all settings in which interacting quantum many-body systems are coupled to dissipative channels, in particular to excitonpolariton condensates [19, 20], ultracold quantum gases in optical cavities [22], and Rydberg polaritons [61].

We acknowledge fruitful discussions with H. P. Büchler, S. Gopalakrishnan, N. Lang, T. Lee, M. Lemeshko, I. Lesanovsky, E. Levi, M. Marcuzzi, and A. Rapp. This work was funded by the Volkswagen Foundation.

* hweimer@itp.uni-hannover.de

[1] W. Kohn, Rev. Mod. Phys. 71, 1253 (1999).

[2] U. Schollwöck, Ann. Phys. 326, 96 (2011).

[3] S. Diehl, A. Micheli, A. Kantian, B. Kraus, H. P. Büchler, and P. Zoller, Nature Phys. 4, 878 (2008).

[4] F. Verstraete, M. M. Wolf, and J. Ignacio Cirac, Nature Phys. 5, 633 (2009).

[5] H. Weimer, M. Müller, I. Lesanovsky, P. Zoller, and H. P. Büchler, Nature Phys. 6, 382 (2010).

[6] S. Diehl, W. Yi, A. J. Daley, and P. Zoller, Phys. Rev. Lett. 105, 227001 (2010).

[7] A. F. Alharbi and Z. Ficek, Phys. Rev. A 82, 054103 (2010).

[8] J. T. Barreiro, M. Müller, P. Schindler, D. Nigg, T. Monz, M. Chwalla, M. Hennrich, C. F. Roos, P. Zoller, and R. Blatt, Nature 470, 486 (2011).

[9] H. Krauter, C. A. Muschik, K. Jensen, W. Wasilewski, J. M. Petersen, J. I. Cirac, and E. S. Polzik, Phys. Rev. Lett. 107, 080503 (2011).

[10] G. Watanabe and H. Mäkelä, Phys. Rev. A 85, 023604 (2012).

[11] D. D. B. Rao and K. Mølmer, Phys. Rev. Lett. 111, 033606 (2013).

[12] A. W. Carr and M. Saffman, Phys. Rev. Lett. 111, 033607 (2013).
[13] H. Weimer, Mol. Phys. 111, 1753 (2013).

[14] J. Otterbach and M. Lemeshko, Phys. Rev. Lett. 113, 070401 (2014).

[15] S. D. Huber and H. P. Büchler, Phys. Rev. Lett. 108, 193006 (2012).

[16] B. Zhao, A. W. Glaetzle, G. Pupillo, and P. Zoller, Phys. Rev. Lett. 108, 193007 (2012).

[17] C. Ates, B. Olmos, W. Li, and I. Lesanovsky, Phys. Rev. Lett. 109, 233003 (2012).

[18] M. Lemeshko and H. Weimer, Nature Commun. 4, 2230 (2013).

[19] J. Kasprzak, M. Richard, S. Kundermann, A. Baas, P. Jeambrun, J. M. J. Keeling, F. M. Marchetti, M. H. Szymaska, R. Andr, J. L. Staehli, V. Savona, P. B. Littlewood, B. Deveaud, and L. S. Dang, Nature 443, 409 (2006).

[20] A. Amo, D. Sanvitto, F. P. Laussy, D. Ballarini, E. d. Valle, M. D. Martin, A. Lematre, J. Bloch, D. N. Krizhanovskii, M. S. Skolnick, C. Tejedor, and L. Via, Nature 457, 291 (2009).

[21] M. J. Hartmann, Phys. Rev. Lett. 104, 113601 (2010).

[22] K. Baumann, C. Guerlin, F. Brennecke, and T. Esslinger, Nature 464, 1301 (2010).

[23] D. Nagy, G. Kónya, G. Szirmai, and P. Domokos, Phys. Rev. Lett. 104, 130401 (2010).

[24] S. Diehl, A. Tomadin, A. Micheli, R. Fazio, and P. Zoller, Phys. Rev. Lett. 105, 015702 (2010).

[25] A. Tomadin, S. Diehl, and P. Zoller, Phys. Rev. A 83, 013611 (2011).

[26] T. E. Lee, H. Häffner, and M. C. Cross, Phys. Rev. A 84, 031402 (2011).

[27] E. M. Kessler, G. Giedke, A. Imamoglu, S. F. Yelin, M. D. Lukin, and J. I. Cirac, Phys. Rev. A 86, 012116 (2012).

[28] M. Höning, M. Moos, and M. Fleischhauer, Phys. Rev. A 86, 013606 (2012).

[29] M. Höning, D. Muth, D. Petrosyan, and M. Fleischhauer, Phys. Rev. A 87, 023401 (2013).

[30] B. Horstmann, J. I. Cirac, and G. Giedke, Phys. Rev. A 87, 012108 (2013).

[31] E. G. D. Torre, S. Diehl, M. D. Lukin, S. Sachdev, and P. Strack, Phys. Rev. A 87, 023831 (2013).

[32] L. M. Sieberer, S. D. Huber, E. Altman, and S. Diehl, Phys. Rev. Lett. 110, 195301 (2013).

[33] J. Qian, L. Zhou, and W. Zhang, Phys. Rev. A 87, 063421 (2013).

[34] T. E. Lee, S. Gopalakrishnan, and M. D. Lukin, Phys. Rev. Lett. 110, 257204 (2013).

[35] C. Carr, R. Ritter, C. G. Wade, C. S. Adams, and K. J. Weatherill, Phys. Rev. Lett. 111, 113901 (2013).

[36] C. Joshi, F. Nissen, and J. Keeling, Phys. Rev. A 88, 063835 (2013).

[37] N. Malossi, M. M. Valado, S. Scotto, P. Huillery, P. Pillet, D. Ciampini, E. Arimondo, and O. Morsch, Phys. Rev. Lett. 113, 023006 (2014).

[38] M. Marcuzzi, E. Levi, S. Diehl, J. P. Garrahan, and I. Lesanovsky, arXiv:1406.1015 (2014).

[39] N. Lang and H. P. Büchler, arXiv:1408.4616 (2014).

[40] H.-P. Breuer and F. Petruccione, The Theory of Open Quantum Systems (Oxford University Press, Oxford, 2002).

[41] I. Pižorn, Phys. Rev. A 88, 043635 (2013).

[42] K. Liu, L. Tan, C.-H. Lv, and W.-M. Liu, Phys. Rev. A 83, 063840 (2011).

[43] T. E. Lee, H. Häffner, and M. C. Cross, Phys. Rev. Lett. 
108, 023602 (2012).

[44] J. Jin, D. Rossini, R. Fazio, M. Leib, and M. J. Hartmann, Phys. Rev. Lett. 110, 163605 (2013).

[45] H. Weimer, M. J. Henrich, F. Rempp, H. Schröder, and G. Mahler, Europhys. Lett. 83, 30008 (2008).

[46] P. Navez and R. Schützhold, Phys. Rev. A 82, 063603 (2010).

[47] See Supplemental Material for a proof of the biasedness of Schatten norms violating the linearity condition, which includes Ref. 48].

[48] R. Bhatia, Matrix analysis, Graduate Texts in Mathematics, Vol. 169 (Springer, New York, 1997).

[49] M. Hoening, W. Abdussalam, M. Fleischhauer, and T. Pohl, Phys. Rev. A 90, 021603 (2014).

[50] R. Löw, H. Weimer, J. Nipper, J. B. Balewski, B. Butscher, H. P. Büchler, and T. Pfau, J. Phys. B 45, 113001 (2012).

[51] A. Hu, T. E. Lee, and C. W. Clark, Phys. Rev. A 88, 053627 (2013).

[52] C. Ates, B. Olmos, J. P. Garrahan, and I. Lesanovsky,
Phys. Rev. A 85, 043620 (2012).

[53] A. J. Daley, Adv. Phys. 63, 77 (2014).

[54] J. Johansson, P. Nation, and F. Nori, Comp. Phys. Comm. 184, 1234 (2013).

[55] M. E. Fisher and A. N. Berker, Phys. Rev. B 26, 2507 (1982).

[56] K. Huang, Statistical Mechanics (John Wiley and Sons, New York, 1987).

[57] G. S. Agarwal and S. R. Shenoy, Phys. Rev. A 23, 2719 (1981).

[58] A. Tomadin, V. Giovannetti, R. Fazio, D. Gerace, I. Carusotto, H. E. Türeci, and A. Imamoglu, Phys. Rev. A 81, 061801 (2010).

[59] T. C. H. Liew and V. Savona, New Journal of Physics 15, 025015 (2013).

[60] A. Le Boité, G. Orso, and C. Ciuti, Phys. Rev. Lett. 110, 233601 (2013).

[61] T. Peyronel, O. Firstenberg, Q.-Y. Liang, S. Hofferberth, A. V. Gorshkov, T. Pohl, M. D. Lukin, and V. Vuletić, Nature 488, 57 (2012). 


\title{
Supplemental Material for "Variational principle for steady states of dissipative quantum many-body systems"
}

\author{
Hendrik Weimer* \\ Institut für Theoretische Physik, Leibniz Universität Hannover, Appelstr. 2, 30167 Hannover, Germany
}

\section{BIASEDNESS OF SCHATTEN NORMS}

We consider Schatten norms of the form

$$
\|\dot{\rho}\|=\operatorname{Tr}\left\{|\dot{\rho}|^{p}\right\} .
$$

with $p \geq 1$. Note that we have defined the norm without taking the $p$ th root of the final result as this does not change the variational minimum. Schatten norms form the most common class of norms invariant under unitary transformations of the form $\dot{\rho} \rightarrow U \dot{\rho} U^{\dagger}$ and corresponds to vector norms of the set of eigenvalues of $\dot{\rho}$. Important examples include the trace norm $(p=1)$, the Hilbert-Schmidt or Frobenius norm $(p=2)$ and the spectral norm $(p=\infty)$. For $p<1$, the expression does not produce a valid norm as the triangle inequality is violated [1]. Here, we consider the consequences of choosing a variational norm that violates the linearity condition, i.e.,

$$
\|\dot{\rho}\| \neq\|\lambda \dot{\rho}\| / \lambda
$$

which is the case for all Schatten norms with $p>1$.

For product states of the form $\rho=\prod_{i} \rho_{i}$, we can write the resulting variational norm as [cf. Eq. (6) of the main text]

$$
\|\dot{\rho}\|=\left\|\sum_{i} \mathcal{R} \dot{\rho}_{i}+\sum_{\langle i j\rangle} \mathcal{R} \dot{C}_{i j}\right\| \equiv\left\|\sum_{\langle i j\rangle} \mathcal{R} A_{i j}\right\|
$$

Now, we are interested in obtaining an upper bound for the norm, which we will then evaluate for the maximally mixed state. We can use the triangle inequality, resulting in

$$
\|\dot{\rho}\| \leq \sum_{\langle i j\rangle}\left\|A_{i j}\right\| \prod_{k \neq i j}\left\|\rho_{k}\right\|
$$

As the sum contains on the order of $N$ terms, we can find a constant $a$ such that

$$
\|\dot{\rho}\| \leq a N \prod_{k>2}\left\|\rho_{k}\right\| .
$$

For the maximally mixed state, this yields

$$
\|\dot{\rho}\| \leq \frac{a N}{d^{(p-1)(N-2)}},
$$

where $d$ is the Hilbert space dimension of a single site. Here, we already see that the trace norm with $p=1$ is special, as it is the only Schatten norm not decaying exponentially with the system size $N$.

Next, we calculate a lower bound for the norm of Eq. (SM-3). Here, it is important to note that the matrices $A_{i j}$ cannot be zero as the true stationary state cannot be described by a product state for finite interactions and finite spatial dimensions 2]. As the $A_{i j}$ act on different parts of the Hilbert space, their sum cannot vanish either and we can find another constant such that

$$
\|\dot{\rho}\| \geq b \prod_{k>2}\left\|\rho_{k}\right\| .
$$

If we choose a variational state different from the maximally mixed state, the largest eigenvalue of $\rho_{k}, p_{\max }$, will satisfy the relation $p_{\max }>1 / d$. Without loss of generality, we assume $p_{\max }$ to be the same for all sites, which yields

$$
\|\dot{\rho}\| \geq b\left[p_{\max }^{p}+\frac{d-2}{d^{p}}+\left(\frac{2}{d}-p_{\max }\right)^{p}\right]^{N-2} .
$$


This expression again describes an exponential decay in $N$ for any $p>1$, but with a strictly smaller base than for the upper bound of Eq. (SM-6) for the maximally mixed state. Consequently, there will be a finite value of $N$ above which the maximally mixed state will always produce a lower norm than any other state, proving the biasedness of Schatten norms with $p>1$.

* hweimer@itp.uni-hannover.de

[1] R. Bhatia, Matrix analysis, Graduate Texts in Mathematics, Vol. 169 (Springer, New York, 1997).

[2] P. Navez and R. Schützhold, Phys. Rev. A 82, 063603 (2010). 